\title{
Supportive Management Resolved a Colonic Intramural Hematoma in an Anticoagulant User
}

\author{
KiWook Kwon, Dae Young Cheung, Yoon Seo, Shin Bum Kim, Kang Nam Bae, \\ Hyun Jin Kim, Jin Il Kim, Soo-Heon Park and Jae Kwang Kim
}

\begin{abstract}
Intramural hematomas of the colon are rare. Intramural hematomas of the gastrointestinal tract often result from blunt trauma, although they may also occur spontaneously in patients taking anticoagulants or those with blood dyscrasia. Affected patients present with abdominal pain and lower gastrointestinal bleeding, as well as occasional signs of intestinal obstruction. The diagnosis is made using colonoscopy and abdominal CT. Resection of the involved bowel segment is accepted as the standard therapy; however, experience with conservative treatment has also been reported. We herein report the case of a 62-year-old woman taking warfarin who was diagnosed with a colonic intramural hematoma and completely recovered with conservative management.
\end{abstract}

Key words: colon, intramural hematoma, warfarin

(Intern Med 53: 1505-1509, 2014)

(DOI: 10.2169/internalmedicine.53.2358)

\section{Introduction}

Colonic intramural hematomas are rare and often associated with blunt trauma, although they can also occur spontaneously in patients taking anticoagulation therapy or those withblood dyscrasia (1-4). Iatrogenic injury during endoscopy has also been reported as a causative factor. Intramural hematomas formed under anticoagulation treatment often involve the small intestine and duodenum, whereas the colon is rarely involved (3-5). We herein report the case of a patient with a warfarin-associated colonic intramural hematoma that developed spontaneously and resolved with conservative treatment. In addition, we also review the pertinent literature with respect to the reported clinical course and outcomes of patients with colonic intramural hematomas.

\section{Case Report}

A 62-year-old woman visited the emergency room presenting with bloody stools and lower abdominal cramping pain. Her symptoms began two hours prior to presentation. She appeared acutely ill, although her vital signs, including body temperature and pulse rate, were stable and within the normal reference ranges. A physical examination revealed mild tenderness in the left lower quadrant without signs of peritoneal irritation. The patient reported no causative events, such as abdominal trauma, excessive enema use and so on. A plain abdominal X-ray showed only a nonspecific bowel gas pattern. The white blood cell count was 10,910/ $\mathrm{mm}^{3}$ (normal range: $4,000-10,500 / \mathrm{mm}^{3}$ ), the hemoglobin level was $10.8 \mathrm{mg} / \mathrm{dL}$ (normal range: $12.6-17.7 \mathrm{mg} / \mathrm{dL}$ ) with a hematocrit level of $33.7 \%$ (normal range: $37.5-51 \%$ ) and a platelet count of $258,000 / \mathrm{mm}^{3}$ (normal range: 140,000$\left.415,000 / \mathrm{mm}^{3}\right)$. The prothrombin time was prolonged to 25.6 seconds (10.6 seconds, control value) and the International Normalized Ratio (INR) was 2.42 (INR normal range: 0.81.2). According to the patient's medical history, she took metformin $(500 \mathrm{mg})$ and simvastatin $(10 \mathrm{mg})$ each day for diabetes mellitus and hyperlipidemia, respectively. In addition, the patient also reported that she had taken warfarin for five years since undergoing aortic and mitral valve replacement surgery for mitral and aortic stenosis. Ticlopidine (250 $\mathrm{mg}$ ), furosemide $(40 \mathrm{mg})$ and spironolactone $(25 \mathrm{mg})$ were co-administered with the warfarin. The dose of warfarin was $5.5 \mathrm{mg}$ per day, and the prothrombin time (PT) INR was 

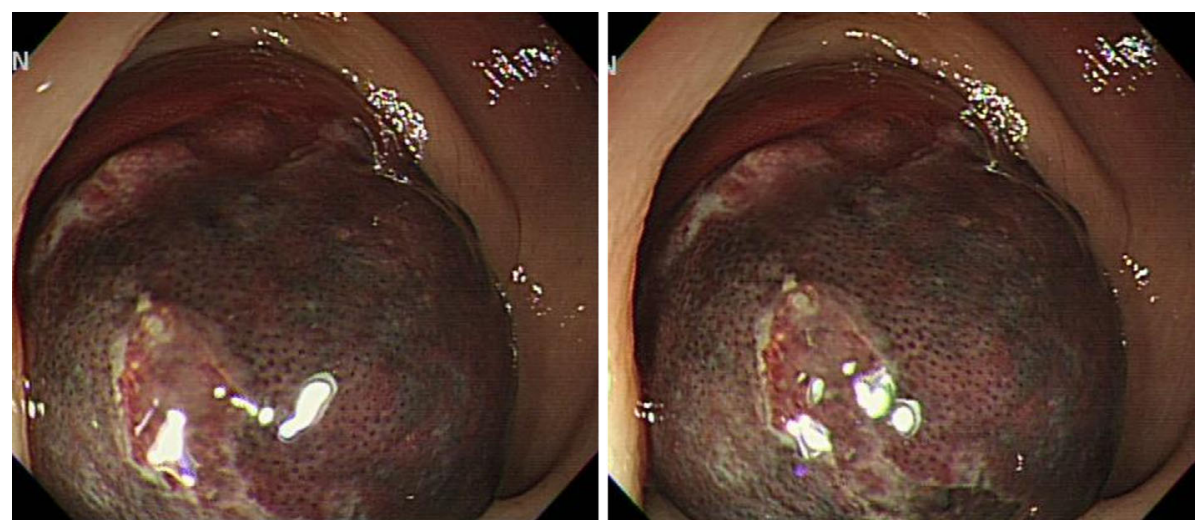

Figure 1. Colonoscopy after bowel preparation showed a colonic intramural hematoma almost completely obstructing the lumen.
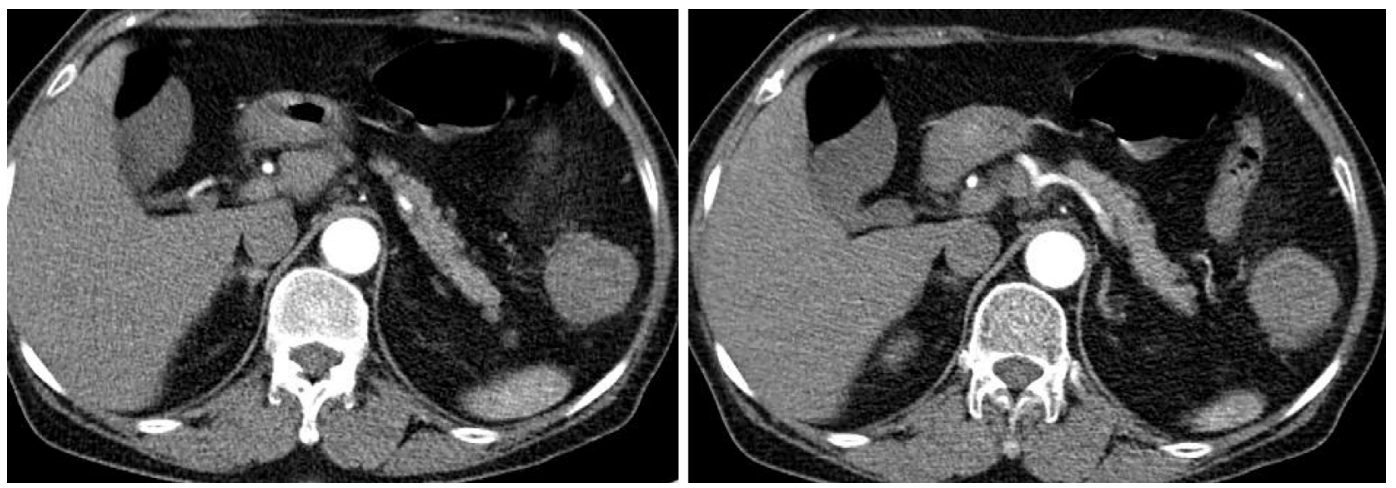

Figure 2. Abdominal CT with contrast enhancement showed no definitive bleeding foci. On a retrospective review, an intraluminal mass with a faint margin was observed in the sigmoid colon.

maintained between 2.0 to 4.0. The warfarin and ticlopidine were discontinued immediately. The patient's colonic bleeding ceased, and her abdominal pain decreased after admission.

A digital rectal examination revealed fresh bloody stool in the rectum without any palpable masses or points of tenderness. Sigmoidoscopy performed immediately showed bloody stools. Clots were found to have occluded the entire rectal lumen, without any definitive bleeding focus. Abdominal CT did not detect any bleeding foci or mucosal or vascular abnormalities (Fig. 1). Simple hydration and volume replacement was administered with crystalloid fluid infusion. Colonoscopy was performed the next day, after bowel preparation. On colonoscopy, a large intramural hematoma was identified in the mid-portion of the sigmoid colon, without bleeding (Fig. 2). The intramural hematoma almost totally occluded the lumen, a colonoscope could not be successfully passed through the region.

Because the patient's bleeding ceased following the discontinuation of warfarin, we decided to treat her conservatively rather than surgically. In order to avoid thrombotic events during the discontinuation of warfarin and ticlopidine, $20 \mathrm{mg}$ of enoxaparin was administered subcutaneously twice daily 48 hours after the cessation of warfarin and ticlopidine. The patient also received intravenous hydra- tion and was allowed to take a sip of water each day for seven days. Follow-up colonoscopy performed on the seventh day revealed the disappearance of the hematoma, leaving a shallow ulcer (Fig. 3). An oral diet was resumed on the seventh day. Warfarin was reintroduced on the 14th day, with enoxaparin treatment overlapped for five days. The PT INR was maintained between 2.0 to 4.0 , and the patient was discharged in a completely recovered state.

\section{Discussion}

Although the major cause of gastrointestinal tract hematomas is abdominal blunt trauma (6), they can develop spontaneously in patients receiving anticoagulation therapy or those with hematologic disease. Among all types of gastrointestinal intramural hematomas, $36 \%$ are believed to be anticoagulation therapy-related (6). Anticoagulation therapy is widely used to treat many conditions, including thrombotic diathesis and atrial fibrillation, and in patients with a history of artificial heart valve replacement. Bleeding is the most serious complication of warfarin treatment, and unwanted bleeding from excess anticoagulation occurs in approximately $7.6 \%$ of patients (4). However, spontaneous hematoma formation in the gastrointestinal tract is uncommon. Small intestinal hematomas are reported in one per 2,500 

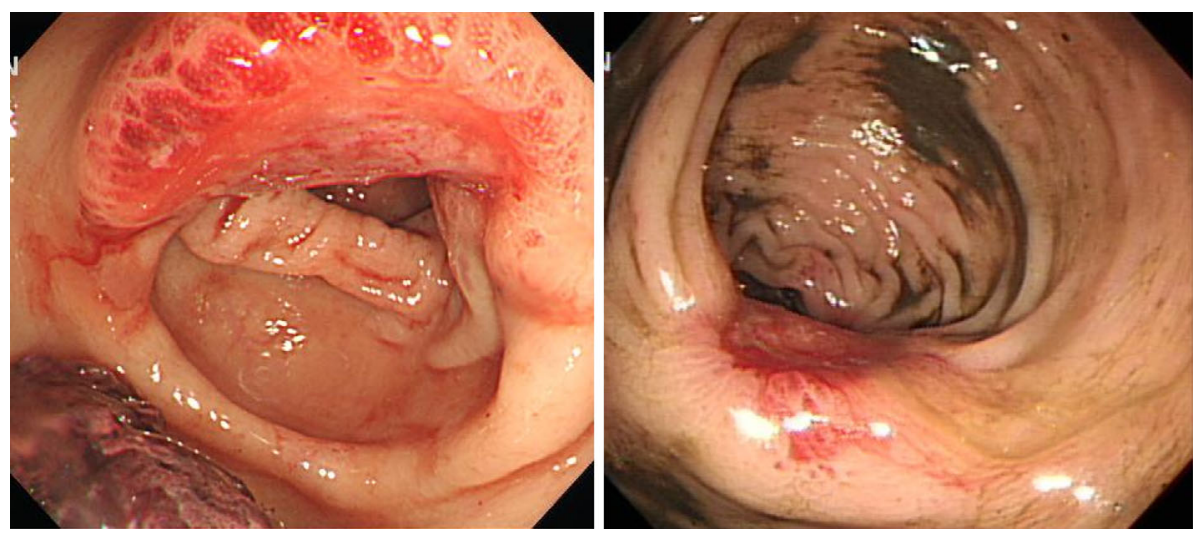

Figure 3. Follow up colonoscopy performed after one week showed a healing ulcer with a shrunken base. The hematoma had detached from the surface and disappeared. The surrounding mucosa appeared healthy without any mucosal abnormalities.

Table. Clinical Characteristics of Colonic Intramural Hematomas in Literatures 1,8-37

\begin{tabular}{cccccccccc}
\hline & \multicolumn{2}{c}{ Sex } & \multicolumn{3}{c}{ Age } & \multicolumn{2}{c}{ Site } & \multicolumn{2}{c}{ Treatment } \\
\hline Cause & Male & Female & $<10$ & $10-50$ & $>50$ & Rectum and S-colon & Other part of colon & Surgery & Medical \\
\hline Anticoagulation- induced(1-8) & 5 & 3 & 0 & 1 & 7 & 6 & 2 & 3 & 5 \\
Trauma & 11 & 0 & 2 & 8 & 1 & 1 & 10 & 6 & 5 \\
Hematologic disease & 4 & 0 & 0 & 2 & 2 & 2 & 2 & 3 & 1 \\
Other reason & 8 & 1 & 2 & 1 & 6 & 6 & 3 & 4 & 5 \\
Total & 28 & 4 & 4 & 12 & 16 & 15 & 17 & 16 & 16 \\
\hline
\end{tabular}

patients on anticoagulation therapy each year, with the duodenum being the most frequently involved site. Intramural colonic hematomas have been described less frequently in the literature (4).

Regarding the possible effects of medications coadministered with warfarin, the initiation of a fibrate or statin inhibiting CYP3A4 enzymes is known to be associated with an increased risk of gastrointestinal bleeding (7). However, in most cases, as in our experience, the risk associated with the co-administration of warfarin and simvastatin can be easily controlled to reduce the incidence of thrombotic and atherosclerotic complications. Pravastatin may possibly be considered a substitute for simvastatin, as this drug is primarily excreted unchanged without CYP3A4 metabolism. Regarding the co-administration of ticlopidine, available guidelines pertaining to the concomitant use of aspirin, a thienopyridine, and warfarin are based on limited trial data and consensus judgment (8). Overall, the selection of combination therapy in patients with cardiovascular disease is considered a matter of clinical judgment based on the physician's perceived balance of the individual patient's risks and benefits.

A review of case reports of colonic intramural hematomas identified 32 cases written in English (Table) (1, 3, 9-37). The mean age of the patients was 42 years, and men were reported more frequently as patients than women (28 to four, respectively). The most common involved sites were the rectum and sigmoid colon. Blunt abdominal trauma was the causative factor in 11 of the 32 cases, and the site of in- jury was frequently in the cecum and/or descending colon. Anticoagulation therapy and hematologic disease were the causative factor in eight and four patients each. Warfarin was the most common causative agent $(1,3,22,29)$, followed by heparin $(21,32)$. On patient treated with warfarin in addition to aspirin (10) and another patient treated with aspirin in addition to clopidogrel (23) were also reported. Other causative factors included complications of hemorrhoidopexy, polypectomy and endoscopic tattooing.

The diagnosis of colonic intramural hematoma is established based on clinical suspicion in patients with abdominal pain, signs of intestinal obstruction and bleeding from the rectum. Barium enemas usually show the "picket fence" and "coiled-spring" signs. Abdominal CT also reveals similar findings; however, they are not specific to cases of intramural hematomas. As in our experience, emergency colonoscopy with an enema can be useful if performed gently by an experienced endoscopist. Colonic intramural hematomas appear round to oval in shape and dark reddish with a submucosal mass obstructing the luminal space. The use of a forceps biopsy can be helpful in cases of suspicion of intraluminal tumors. However, the tissue contained in hematomas is very friable and easily fragmented.

The management of intramural colonic hematomas consists of treatments to control bleeding and resolve the hematoma. Control of bleeding is commonly achieved with the immediate cessation of anticoagulants and, if needed, the administration of vitamin $\mathrm{K}$ and fresh frozen plasma to rapidly correct bleeding diathesis. In order to resolve the hema- 


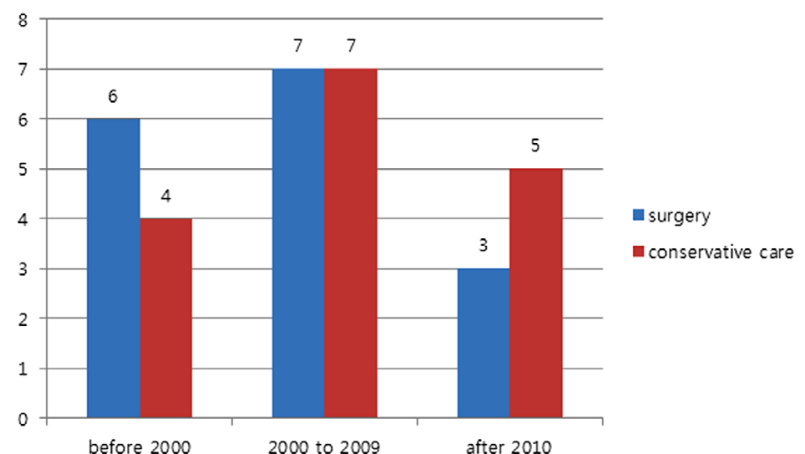

Figure 4. Changes in treatment modalities according to the decade

toma and its clinical consequences, such as colonic obstruction, surgical resection was accepted as the standard of treatment in the past. Before 2000, surgery was performed in six cases, and conservative care was performed in four cases. However, in many cases, conservative care has become the treatment of choice. In 2010, surgery was performed in only three of all eight cases reported (Fig. 4). Endoscopic drainage and removal can be employed in selected patients (20). In our literature review, surgical resection was employed as a treatment modality in $16(50 \%)$ of the 32 patients. Three of eight patients on anticoagulation therapy underwent surgical resection because the hematoma did not resolve with conservative treatment and later relapsed following the resumption of anticoagulation therapy. Six of the 11 trauma victims and three of the four patients with hematologic disorders were reported to have received surgical treatment for reasons such as the uncertainty of diagnosis and unstable vital status. The remaining four patients received surgical treatment due to massive bleeding and an uncertain diagnosis(Fig. 5). As in our experience, it is reasonable to administer medical treatment first if the patient's vital signs are stable. Surgical treatment should be reserved for patients with an uncertain diagnosis and/or those who exhibit signs of intestinal necrosis or peritonitis or a deteriorating condition despite medical treatment (38).

The strategy for treating bleeding complications in patients receiving anticoagulants for cardiovascular disease varies depending on the physician and institute. The duration of anticoagulation cessation should be determined for each individual patient based on their clinical condition and be minimized as much as possible. Low-molecular-weight heparin can be a substitute for warfarin in bleeding patients with cardiovascular disease, and we used enoxaparin after two days of discontinuation of warfarin and ticlopidine.

In summary, spontaneous colonic intramural hematoma formation is an uncommon complication in patients receiving excessive anticoagulation therapy with warfarin. When patients on warfarin anticoagulation with an excessively prolonged PT INR present with unexplained abdominal pain and bleeding, the presence of gastrointestinal intramural or submucosal hematomas should be included in the differen-

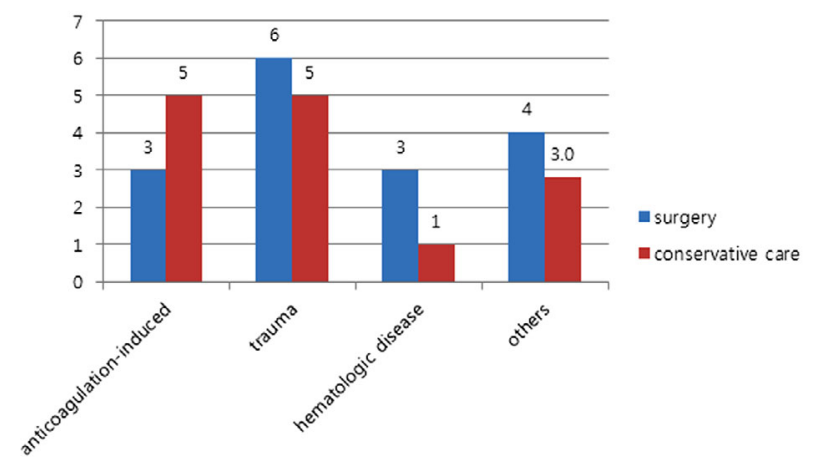

Figure 5. Differences intreatment decisions according to the cause of the colonic intramural hematoma

tial diagnosis. Providing early recognition and prompt management of the disease is crucial for avoiding unwanted surgery and enabling complete resolution with conservative treatment.

The authors state that they have no Conflict of Interest (COI).

\section{References}

1. TerKonda SP, Nichols FC 3rd, Sarr MG. Spontaneous perforating hematoma of the rectum. Report of a case. Dis Colon Rectum 35: 270-272, 1992.

2. Chaiteerakij R, Treeprasertsuk S, Mahachai V, et al. Anticoagulant-induced intramural intestinal hematoma: report of three cases and literature review. J Med Assoc Thai 91: 12851290, 2008.

3. Krysa J, Shahabdeen M, South LM. A case of acute large bowel obstruction, presenting in a patient taking warfarin. Emerg Med J 20: E8, 2003.

4. Polat C, Dervisoglu A, Guven $\mathrm{H}$, et al. Anticoagulant-induced intramural intestinal hematoma. Am J Emerg Med 21: 208-211, 2003.

5. Fischer J, Samson P, Robertson G. Anticoagulant-induced intramural haematoma of the caecum mimicking a colonic tumour. $\mathrm{N} \mathrm{Z}$ Med J 123: 75-78, 2010.

6. Hughes CE 3rd, Conn J Jr, Sherman JO. Intramural hematoma of the gastrointestinal tract. Am J Surg 133: 276-279, 1977.

7. Schelleman H, Bilker W, Brensinger C, et al. Fibrate/Statin initiation in warfarin users and gastrointestinal bleeding risk. Am J Med 123: 151-157, 2010.

8. Hermosillo AJ, Spinler SA. Aspirin, clopidogrel, and warfarin: is the combination appropriate and effective or inappropriate and too dangerous? Ann Pharmacother 42: 790-805, 2008.

9. Augustin G, Smud D, Kinda E, et al. Intra-abdominal bleeding from a seromuscular tear of an ascending rectosigmoid intramural hematoma after stapled hemorrhoidopexy. Can J Surg 52: E14E15, 2009.

10. Bakaeen FG, Chow M, Ghadir M, et al. Ruptured colonic intramural hematoma with massive hemorrhage after aortic valve replacement. Am Surg 76: 647-648, 2010.

11. Calabuig R, Ortiz C, Sueiras A, et al. Intramural hematoma of the cecum: report of two cases. Dis Colon Rectum 45: 564-566, 2002.

12. Calenoff L, Lounsbury F. Intramural hematoma of the sigmoid. Am J Roentgenol Radium Ther Nucl Med 107: 170-174, 1969.

13. De Santis G, Gola P, Lancione L, et al. Sigmoid intramural hematoma and hemoperitoneum: an early severe complication after stapled hemorrhoidopexy. Tech Coloproctol 16: 315-317, 2012. 
14. Harrison HC, Lord RS, Chesterman CN, et al. Spontaneous intramural haematoma in the sigmoid colon of a haemophiliac. Aust $\mathrm{N}$ Z J Surg 42: 69-70, 1972.

15. Hou MM, Tsou YK. Education and imaging. Gastrointestinal: Acute colonic intramural hematoma after blunt abdominal trauma. J Gastroenterol Hepatol 24: 494, 2009.

16. Jarry J, Biscay D, Lepront D, et al. Spontaneous intramural haematoma of the sigmoid colon causing acute intestinal obstruction in a haemophiliac: report of a case. Haemophilia 14: 383-384, 2008.

17. Jeffrey RB, Federle MP, Stein SM, et al. Case report. Intramural hematoma of the cecum following blunt trauma. J Comput Assist Tomogr 6: 404-405, 1982.

18. Kao CL, Lu MS, Chang JP. Acute type A intramural hematoma in a patient with substernal colon interposition. Tex Heart Inst J 38: 454-455, 2011.

19. Karjoo M, Domachowske J, Trust S. Intramuiral hematoma of the descending colon after blunt abdominal trauma. Clinical Pediatrics 39: 373-374, 2000.

20. Kwon CI, Ko KH, Kim HY, et al. Bowel obstruction caused by an intramural duodenal hematoma: a case report of endoscopic incision and drainage. J Korean Med Sci 24: 179-183, 2009.

21. Lee $\mathrm{SH}$, Lee JH, Park DH, et al. Intramural colonic hematoma: complication of anticoagulation with heparin. Gastrointest Endosc 62: 783-784, 2005.

22. Li ZL, Wang ZJ, Han JG. Spontaneous perforation of an intramural rectal hematoma: report of a case. World J Gastroenterol 18: 2438-2440, 2012.

23. Liu Y, Yang S, Tong Q. Spontaneous intramural hematoma of colon. Clin Gastroenterol Hepatol 10: e38, 2012.

24. Marques I, Lagos AC, Pinto A, et al. Rectal intramural hematoma: a rare complication of endoscopic tattooing. Gastrointest Endosc 73: 366-367, 2011.

25. McClenathan JH, Dabadghav N. Blunt rectal trauma causing intramural rectal hematoma: report of a case. Dis Colon Rectum 47: 380-382, 2004.

26. Nakayama Y, Fukushima M, Sakai M, et al. Intramural hematoma of the cecum as the lead point of intussusception in an elderly patient with hemophilia A: report of a case. Surg Today 36: 563565,2006

27. Nance FC, Crowder VH. Intramural hematoma of the colon following blunt trauma to the abdomen. Am Surg 34: 85-87, 1968.

28. Nogales Rincon O, Yepes Barreto I, Hernando Alonso A, et al. Large intramural colonic hematoma after polypectomy. Endoscopy 41 (Suppl 2): E3, 2009.

29. Pates DR SR, Hand G. Intestinal obstruction due to intramural hematoma of the colon, a complication of sodium warfarin therapy: report of a case. Dis Colon Rectum 16: 416-418, 1973.

30. Sachdeva RS, Jaeger A, Norton K, et al. Intramural hematoma of the transverse colon in battered child syndrome. J Pediatr Gastroenterol Nutr 18: 111-113, 1994.

31. Schiller M, Morse TS, Frye TR. Severe rectal bleeding from an intramural hematoma of the sigmoid. Pediatrics 48: 146-148, 1971.

32. Shankarnaryanan $\mathrm{S}$, Hardikar W. Unusual cause of neonatal rectal bleeding: colonic intramural haematoma. J Paediatr Child Health 48: E108-E109, 2012.

33. Umeda I, Ohta H, Doi T, et al. Idiopathic intramural hematoma of the colon. Gastrointest Endosc 66: 861-864, 2007.

34. Welling RE, Reilly PS. Nonoperative treatment of a traumatic intramural hematoma of the ascending colon. South Med J 79: 1309-1310, 1986.

35. Westcott JL, Smith JR. Mesentsery and colon injuries secondary to blunt trauma. Radiology 114: 597-600, 1975.

36. Yin WY GM, Huang SM, Chen HT, Chang TM. Acute colonic intramural hematoma due to blunt abdominal trauma. Int Surg 85: $51-54,2000$

37. Howard JM, Hanly AM, Stephens RB. Conservative management resulting in complete resolution of a double intussusception in an adult haemophiliac. Colorectal Dis 10: 197-198, 2008.

38. Lobo L, Koudki R, Prasad Hl K, et al. Colon obstruction due to an anticoagulant induced intramural haematoma; a rare case report. J Clin Diagn Res 7: 739-741, 2013.

(C) 2014 The Japanese Society of Internal Medicine http://www.naika.or.jp/imonline/index.html 